\title{
Pharmacodynamic Effects and Pharmacokinetic Profiles of Keto-Doxapram and Doxapram in Newborn Lambs ${ }^{1}$
}

\author{
A. BAIRAM ${ }^{2}$, P. W. BLANCHARD, K. MULlAHOO, K. BEHARRY, N. LAUDIGNON, AND \\ J. V. ARANDA \\ Jeremy Rill Apnea Research Network; Centre de Recherche, du CHUL et HSFA, Département de Pédiatrie, \\ Services de néonatologie, Université Laval, Québec City; Newborn Medicine Service, and Developmental \\ Pharmacology, McGill University, Montreal, Canada; and Unité INSERM 272, Nancy, France
}

\begin{abstract}
Keto-doxapram (keto-dox), an oxidative metabolite of doxapram, is a possible ventilatory stimulating agent. Our study characterizes its ventilatory properties, pharmacodynamic effects, and pharmacokinetic profile, and those of its parent compound, doxapram. Two groups of five awake, unsedated, newborn lambs (2- to 6-d old) received, respectively, i.v. infusions of keto-dox or doxapram $(2.5 \mathrm{mg} / \mathrm{kg})$ over a period of $1 \mathrm{~min}$. Ventilatory parameters were continuously recorded before and for $1 \mathrm{~h}$ after the drug infusion. The pharmacokinetic profiles of both drugs were determined from blood samples collected serially before and after drug injection. Both drugs stimulated ventilation. Keto-dox increased baseline minute ventilation by $46 \pm 6.1 \%$ and $27.8 \pm 8.1 \%(p<0.002)$ at 1 and $5 \mathrm{~min}$, respectively, an effect that decreased after 5 min of infusion. Doxapram increased minute ventilation by $57 \pm 9 \%(p<0.002)$ at $1 \mathrm{~min}$, and by $48 \pm 7 \%$ at $5 \mathrm{~min}$, but its effect lasted for $20 \mathrm{~min}$ after injection. Compared with the effects of keto-dox, this doxapram increase was significantly higher $(p<0.02)$. Also, doxapram, but not keto-dox, caused an increase in systolic blood pressure (from $110 \pm 3.5$ to $118 \pm 3.4 \mathrm{~mm} \mathrm{Hg}$ at $10 \mathrm{~min}, p<0.01$ ), as well as a change in neuro-behavior. Both drugs exhibited a biexponential decay curve, characterized by a short alpha and a longer beta $t_{1 / 2}$, but keto-dox has a faster elimination rate. These data demonstrate that both doxapram and ketodox have respiratory-stimulating properties but that only doxapram is associated with adverse effects such as increased blood pressure and agitation. Keto-dox, however, has a shorter beta $t_{1 / 2}$ than doxapram. In addition, doxapram is biotransformed to keto-dox, although the converse does not happen. The results suggest that further studies on the use of keto-dox as a therapeutic agent are warranted. (Pediatr Res 28: 142-146, 1990)
\end{abstract}

\section{Abbreviations}

\section{f, respiratory rate}

keto-dox, keto-doxapram

$\mathrm{PaCO}_{2}$, partial pressure of $\mathrm{CO}_{2}$ in arterial blood

Received July 31, 1989: accepted March 29, 1990

Correspondence and reprint requests: Dr. Pierre W. Blanchard, Director, Division of Neonatology, Department of Pediatrics, Laval University, CHUL Research Center, 2705, Laurier Blvd.. Québec City, Québec, CANADA G1V 4G2.

Supported by the Quebec Lung Association and the Medical Research Council of Canada (MRC 10219).

Presented in part at the Society for Pediatric Research annual meeting. Washington D.C., May 1989.

Current address: Maternité régionale de Nancy, Service de néonatologie, rue du Docteur Heydenreich, 54042 Nancy. France. Dr. A. Bairam was a Research Fellow of the Pollack Foundation, Division of Neonatology, Sir Mortimer B. DavisJewish General Hospital, Montreal, at the iime of this study.

\author{
$\mathrm{PaO}_{2}$, partial pressure of $\mathrm{O}_{2}$ in arterial blood \\ VE, minute ventilation \\ $\mathrm{Vt}$, tidal volume \\ $\mathrm{Vt} / \mathrm{Ti}$, mean inspiratory flow
}

Doxapram is a respiratory stimulant (1) with a high selective activity on respiratory neurons (2) and a demonstrated efficacy in the treatment of premature neonates with idiopathic apnea, especially those resistant to methylxanthine, theophylline, or caffeine (3-6). Doxapram is mainly metabolized by oxidation (7), with keto-dox (AHR 5955) being its main oxidative product in adults and newborns $(8,9)$. Whether this oxidative metabolite has any pharmacologic activity is not yet known. Aranda et al. (9) have, in a preliminary study in premature neonates, suggested that keto-dox is an active metabolite, one partly responsible for the respiratory effect of doxapram. The question, however, is whether the rapid disappearance of the effects of doxapram, in less than $1 \mathrm{~h}$ after cessation of therapy (6), may, in fact, be related to its biotransformation to keto-dox, which itself may have very short pharmacologic effects.

Our study was designed 1 ) to determine whether keto-dox possesses respiratory stimulant activities, 2) to identify its respiratory activities and potential side effects related to doxapram, and 3 ) to determine the pharmacokinetics profile of both ketodox and doxapram.

\section{SUBJECTS AND METHODS}

Experimental design. Two groups of 2- to 6-d-old newborn lambs were studied. The first group consisted of five newborn lambs. Using a crossover design, each lamb in this first group received either keto-dox dissolved in methanol, or methanol alone, and then the reverse, at 48-h intervals. That is, we injected, at random, three lambs with first the keto-dox dissolved in methanol and, then, $48 \mathrm{~h}$ later, the methanol alone. Two other lambs received the reverse protocol: first methanol alone, then, $48 \mathrm{~h}$ later, keto-dox dissolved in methanol. For the keto-doxplus-methanol protocol, the keto-dox powder (A. H. Robins, Richmond, VA) was dissolved in methanol $(0.8 \mathrm{~mL}$ methanol/ $15 \mathrm{mg}$ of keto-dox) and then diluted with saline to a final volume of $3 \mathrm{~mL}$, before being administered at a dose of $2.5 \mathrm{mg} / \mathrm{kg}$. For the methanol-alone protocol, the methanol was diluted with saline to obtain the same volume of injection as that of the ketodox. Both the keto-dox and methanol solutions were given as bolus i.v. infusions over a period of $1 \mathrm{~min}$. This particular crossover design was chosen to eliminate any effect of methanol per se (an unusual solvent for medication) on respiration, whether that of depression or stimulation of ventilation. 
The second group consisted of five different newborn lambs. They were studied in the same conditions, via a procedure similar to that followed with the first group. One wk after the first study on keto-dox was completed, these lambs received an i.v. bolus infusion of doxapram at a similar dose of $2.5 \mathrm{mg} / \mathrm{kg}$, again over 1 min. We used a commercial i.v. solution of doxapram (doxapram hydrochloride, $20 \mathrm{mg} / \mathrm{mL}$ preserved in $0.5 \%$ chlorbutanol; A. H. Robins, Montreal, Quebec, Canada). Because the usual commercial preparation of doxapram in Canada already contains chlorbutanol, no comparison study was done between these two compounds.

Animal preparation and method of measurement. On the day of the experiment, two catheters were inserted in each animal. The venous catheter, used for drug injection, was percutaneously introduced into a vessel of one posterior limb. The second, an arterial line used to measure blood pressure and obtain blood samples, was introduced through the left axillary artery via a cutdown under xylocaine local anesthesia. Rectal temperature of each lamb was also recorded.

An adjustable face mask (dead space 2-3 mL) was fitted to each animal. The face mask was attached to a size 0 pneumotachograph (HP 21070 B, Hewlett-Packard Co., Palo Alto, CA). The pneumotachograph flow signal, integrated to give volume by a respiratory integrator (HP $8815 \mathrm{~A}$ ), was recorded on a stripchart recorder (HP 7754 B). The flow signal was simultaneously stored on a computer (Digital Equipment Corp., Marlboro, MA, PDP-11/03) for analysis of each breath. This method, in current use in our laboratory, has been reported in detail elsewhere (1012). The respiratory parameters derived by the computer from each breath were: $\mathrm{VE}, \mathrm{Vt}, \mathrm{f}$, inspiratory time, expiratory time, total respiratory cycle duration, $\mathrm{Vt} / \mathrm{Ti}$ and inspiratory duty cycle.

Study procedures. The conscious lamb, wearing a face mask and breathing room air, was gently restrained on a cart and given time to adjust to its position (that is, until quiet breathing was achieved). The lambs were studied in a presumed awake state because the animals had their eyes open most of the time during recording. The data collection started with a 5 -min baseline recording of respiratory and blood pressure measurements, but was switched at the beginning of the drug injection, and for the subsequent $1-\mathrm{h}$ period, to a continuous recording protocol.

Arterial blood gases for $\mathrm{pH}, \mathrm{PaO}_{2}$ and $\mathrm{PaCO}_{2}$ analysis were drawn before and at the 1-, 10-, 20-, 30-, and 60-min marks after the drug injection. The blood gas values were corrected for the body temperature of the animal.

For all ventilatory variables studied, a number of means were calculated for analysis: the mean of $5 \mathrm{~min}$ of baseline recording; the mean during the minute of drug injection; and the mean of each 5 -min epoch from 1 to 30 and from 50 to $60 \mathrm{~min}$. All were calculated by the computer and used for further analysis. A comparison of the ventilatory data obtained between the ketodox and doxapram was also done at the end of the study.

Because it has been reported that doxapram could induce side effects like increased blood pressure, agitation, and irritability (3, 13), the means of the systolic blood pressures of 1) $5 \mathrm{~min}$ of baseline recording, and 2) each 10 -min epoch from 1 to $30 \mathrm{~min}$, and also from 50 to $60 \mathrm{~min}$ were calculated; we also constantly noted subjective modifications in the animal's normal behavior, such as agitation and increased bleating, before and after injection.

Plasma doxapram and keto-dox were measured by the HPLC method (14) from blood samples collected serially 1 ) at the point of preinjection, and 2) at 1, 10, 20, 30, 45, and $60 \mathrm{~min}$, and subsequently at $2,6,12$, and $24 \mathrm{~h}$ after the injection. The results from both groups were compared at the end of the study.

Data analysis. The results are expressed as the mean \pm SD for the five lambs in each group studied (keto-dox, methanol, and doxapram) and for each variable measured. Data pertaining to $\mathrm{VE}$ and $\mathrm{Vt} / \mathrm{Ti}$ were corrected for body weight.

Pharmacokinetic analysis. The kinetic analysis was done assuming a two open compartment model, and based on the area under the curve (AUC), which is model independent. The elimination rate constant (kel) was calculated using the equation: kel $=$ dose/AUC. The apparent volume of distribution $(\mathrm{AVd})$ was calculated using the equation: $\mathrm{AVd}=$ dose $/ \mathrm{AUC} \times$ kel. Clearance $(\mathrm{CL})$ was calculated using the equation: $\mathrm{CL}=\mathrm{AVd} \times \mathrm{kel}$. The $t_{1 / 2}$ was calculated using the equation: $t_{1 / 2}=0.693 / \mathrm{kel}$.

Statistical analysis. The statistical analysis was done using a two-way analysis of variance for repeated measures within and between groups. The $\mathrm{T}$ method was then used, if a significant difference was found (15). A probability of less than 0.05 was considered significant.

\section{RESULTS}

Pharmacologic drug response. Table 1 shows the baseline data for the different variables measured and for all groups studied. The lambs from the doxapram group had a higher baseline ventilation (at the limit of statistical significance) than the ketodox group; for this reason, results comparing these two groups are expressed as a percent change in ventilation. Figures 1-4 represent the effects of the drugs on ventilation.

Group 1: Methanol Versus Keto-dox. Methanol. In the animals that received the methanol-only injection, none of the variables studied (ventilation, blood gases, blood pressure) showed any change. All the lambs remained quiet, conscious, and awake during the experiment, suggesting that the methanol had not affected their behavior. Incidentally, the amount of methanol used in the keto-dox-methanol solution was far from the amount that could produce a toxic effect (16).

Keto-dox. The keto-dox $(2.5 \mathrm{mg} / \mathrm{kg})$ produced, within $1 \mathrm{~min}$ of injection, an increase in both VE [from $592 \pm 85$ to $863 \pm$ $150 \mathrm{~mL} / \mathrm{kg} / \mathrm{min}(p<0.001)$, Fig. 1] and Vt/Ti [from $23.1 \pm 2.7$ to $34 \pm 5.1 \mathrm{~mL} / \mathrm{kg} / \mathrm{s}(p<0.001)$, Fig. 2], representing a 46 and $47 \%$ increase, respectively. These changes in ventilation were caused mainly by an increase in the lambs' respiratory rate (from $58.0 \pm 9.2$ to $78.5 \pm 15.4$ breaths $/ \mathrm{min}, p<0.005)$, as well as a slight increase in $\mathrm{Vt}$ (from $10.2 \pm 1.3$ to $11.6 \pm 1.3 \mathrm{~mL} / \mathrm{kg}, p<$ 0.01 ). Five $\mathrm{min}$ later, $\mathrm{VE}$ decreased to $743 \pm 105 \mathrm{~mL} / \mathrm{kg} / \mathrm{min}$, whereas Vt/Ti decreased to $29.4 \pm 4.9 \mathrm{~mL} / \mathrm{kg} / \mathrm{s}$; both, however, were still significantly higher than the baseline figures $(p<$ 0.001 ). Within $20 \mathrm{~min}$ of the injection, ventilation had returned to baseline ventilation values. The inspiratory duty cycle did not change significantly. At $1 \mathrm{~min}$ after injection only, the increase in ventilation was accompanied by a decrease in $\mathrm{PaCO}_{2}$ from $36.2 \pm 4.2$ to $30.3 \pm 4.9 \mathrm{~mm} \mathrm{Hg}(4.8 \pm 0.6$ to $4.0 \pm 0.7 \mathrm{kPa}, p$ $<0.002)$. No change in blood pressure nor in the animals behavior was noted after the keto-dox injection.

Group 2: doxapram alone. The ventilatory responses produced with $2.5 \mathrm{mg} / \mathrm{kg}$ of doxapram had a pattern similar to that observed after the keto-dox injection. Within a minute of injection, there was an immediate increase in VE (from $737 \pm 38$ to $1149 \pm 119 \mathrm{~mL} / \mathrm{kg} / \mathrm{min}, p<0.001$ ), and in Vt/Ti (from $25.2 \pm$ 2.4 to $41 \pm 8.5 \mathrm{~mL} / \mathrm{kg} / \mathrm{s}, p<0.001$ ); these increases represented an augmentation of 56 and $63 \%$, respectively (Figs. 3 and 4).

The increase in ventilation with doxapram was essentially due to an increase in $\mathrm{f}$ (from $48.8 \pm 16.1$ to $95.7 \pm 19.7$ breaths/ min, $p<0.001$ ), whereas the $\mathrm{Vt}$ decreased (from $15.1 \pm 4.2$ to $12.0 \pm 2.4 \mathrm{~mL} / \mathrm{kg}, p<0.01)$. These ventilatory responses induced a decrease in $\mathrm{PaCO}_{2}$ from $35.6 \pm 4.0$ to $29.7 \pm 1.1 \mathrm{~mm} \mathrm{Hg}(4.7$ \pm 0.5 to $3.9 \pm 0.1 \mathrm{kPa}, p<0.01)$. The inspiratory duty cycle remained unchanged. At $20 \mathrm{~min}$, ventilation was $953 \pm 121 \mathrm{~mL} /$ $\mathrm{kg} / \mathrm{min}$ for $\mathrm{VE}$, and $29.6 \pm 5.1 \mathrm{~mL} / \mathrm{kg} / \mathrm{s}$ for $\mathrm{Vt} / \mathrm{Ti}$ (for both, $p$ $<0.01$ versus baseline). Ventilation returned almost to baseline value at $30 \mathrm{~min}$ postinjection.

Doxapram ventilatory effects were associated with a transient increase in systolic blood pressure (from $110.0 \pm 7.8$ to $118.0 \pm$ $7.6 \mathrm{~mm} \mathrm{Hg}$, noted at $10 \mathrm{~min}$ only, $p<0.01)$. An obvious subjective change in the animals' behavior manifested itself: agitation occurred and bleating was increased in each animal, lasting for about $10 \mathrm{~min}$ postinjection. 
Table 1. Baseline ventilatory data and blood gas values*

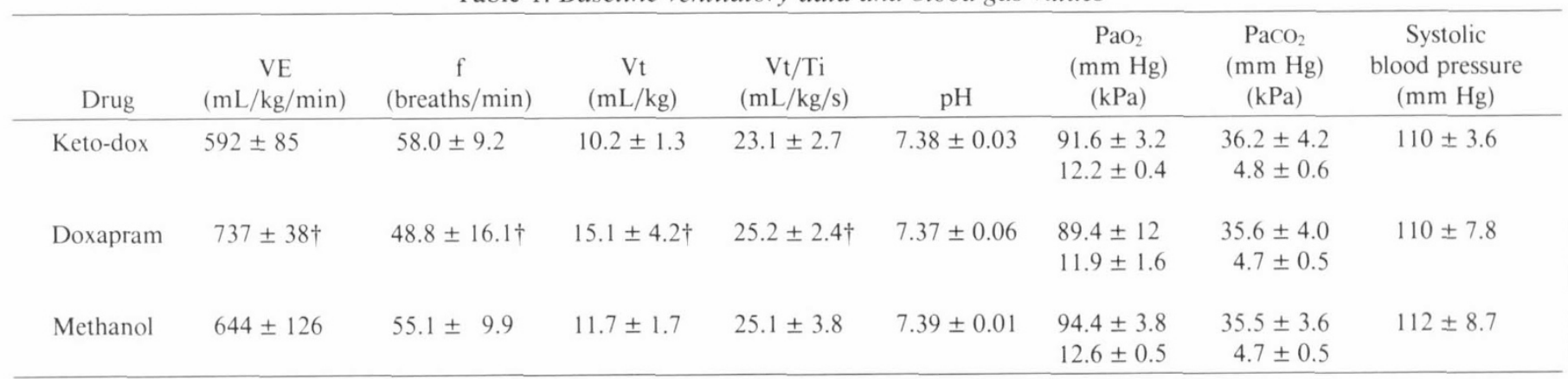

$*$ Values are mean \pm SD for five lambs in each group.

$\dagger p=0.05$ if compared with keto-dox.

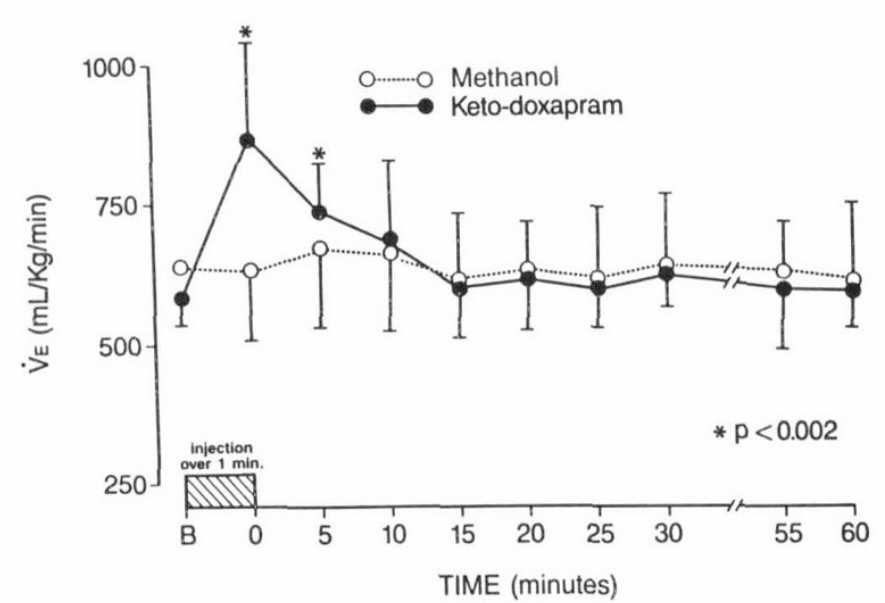

Fig. 1. Effect of keto-dox and methanol on VE. Values are mean \pm $\mathrm{SD}(n=5$ lambs in each group). Asterisks indicate a significant difference from baseline. Closed circles with solid line represent keto-dox, open circles with broken line represent methanol.

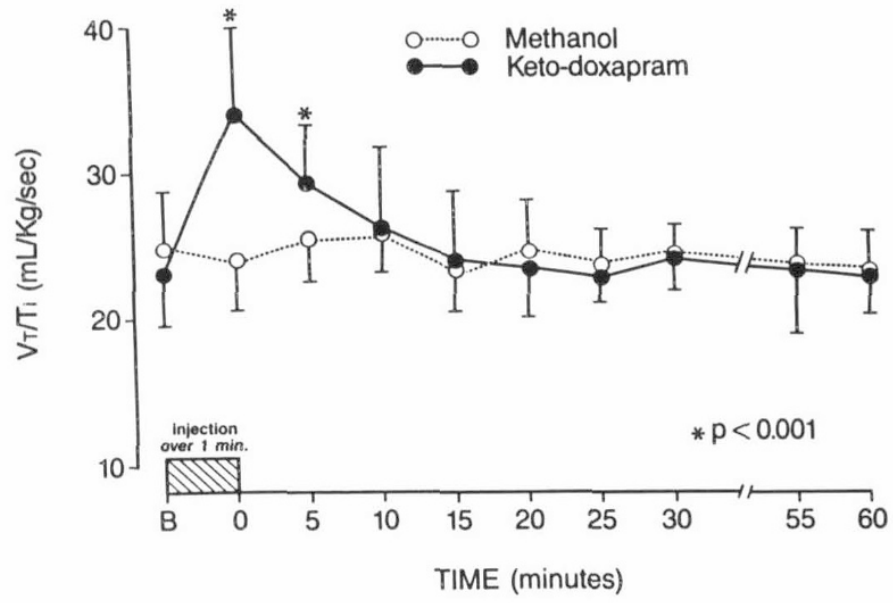

Fig. 2. Effect of keto-dox and methanol on Vt/Ti. Values are mean $\pm \mathrm{SD}(n=5$ lambs in each group). Asterisks indicate a significant difference from baseline. Closed circles with solid line represent ketodox. Open circles with broken line represent methanol.

Comparison of respiratory response: keto-dox versus doxapram. The respiratory stimulation induced by doxapram was greater in both magnitude and duration of action (20 min versus $5 \mathrm{~min}$ ) than that induced by keto-dox with the same size dose. Within the first min of injection, doxapram had increased VE and Vt/Ti by $56 \pm 18$ and $63 \pm 27 \%$, respectively (Figs. 3 and $4)$. These changes were significantly higher $(p<0.01)$ than those brought about by keto-dox, which induced an increase in VE of $46 \pm 13 \%$ and in $\mathrm{Vt} / \mathrm{Ti}$ of $47 \pm 13 \%$. These differences in

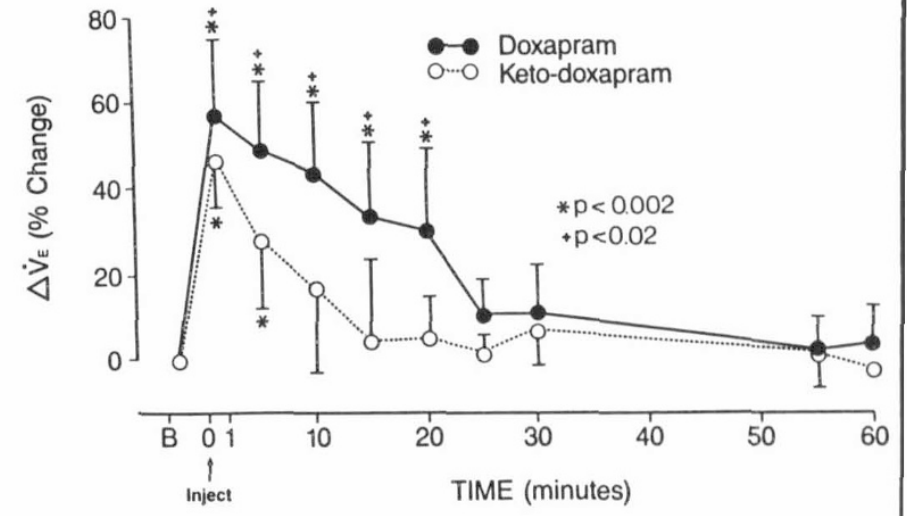

Fig. 3. Effect of keto-dox and doxapram on VE. Values are mean \pm $\mathrm{SD}$ ( $n=5$ in each group) expressed as percent changes in VE from baseline after infusion of the drugs. Asterisks indicate a significant difference from baseline and crosses denote a significant difference between doxapram and keto-dox. Open circles with broken line represent keto-dox, closed circles with solid line represent doxapram.

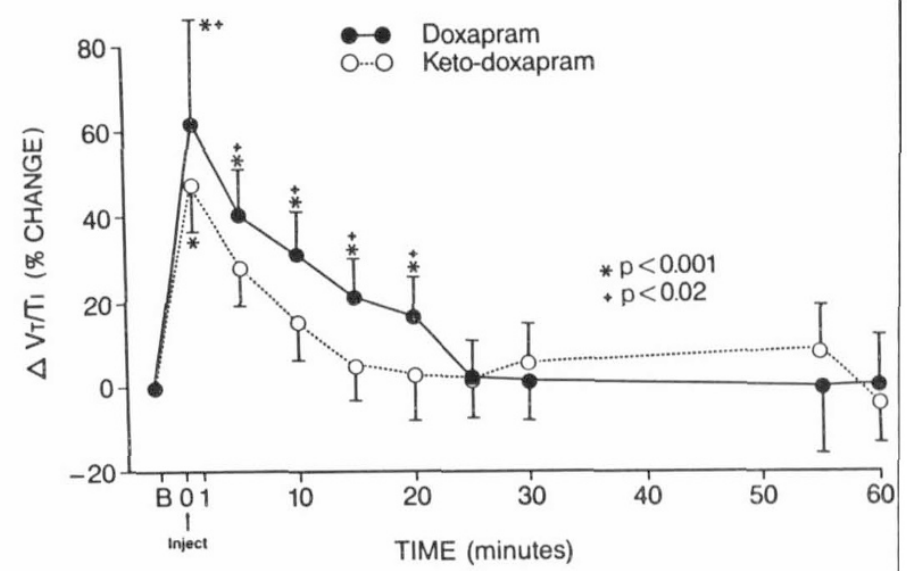

Fig. 4. Effect of keto-dox and doxapram on Vt/Ti. Values are mean $\pm \mathrm{SD}$ ( $n=5$ in each group) expressed as percent changes in Vt/Ti from baseline after drug infusion. Asterisks indicate a significant difference from baseline and crosses denote a significant difference between doxapram and keto-dox. Open circles with broken lines represent keto-dox; closed circles with solid line represent doxapram.

ventilatory responses between the two drugs remained significant until the 20-min mark (Figs. 3 and 4).

Kinetic study (Figs. 5 and 6). Those animals receiving methanol had no detectable doxapram nor keto-dox in their blood samples. Nor did the animals receiving the keto-dox have any detectable doxapram in their samples during the $24 \mathrm{~h}$ after drug injection. In the doxapram group (Fig. 6), however, the metabolite keto-dox generated from doxapram appeared at $20 \mathrm{~min}$ 


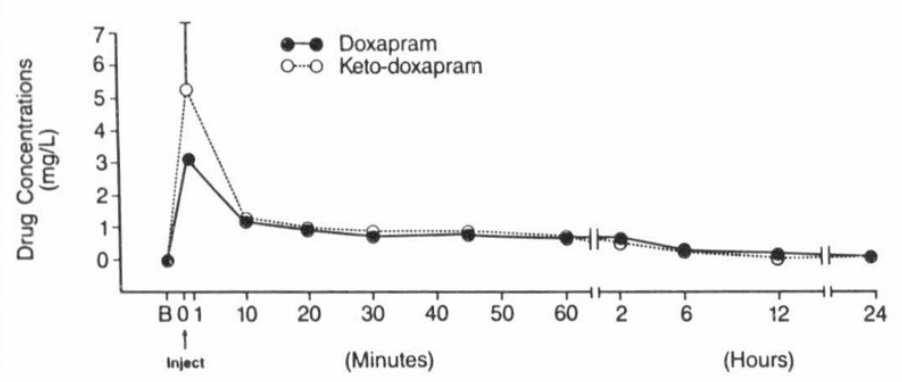

Fig. 5. Plasma drugs concentration of keto-dox and doxapram. Values are the mean $\pm \mathrm{SD}$ of 5 lambs in each group after injection of either doxapram or keto-dox.

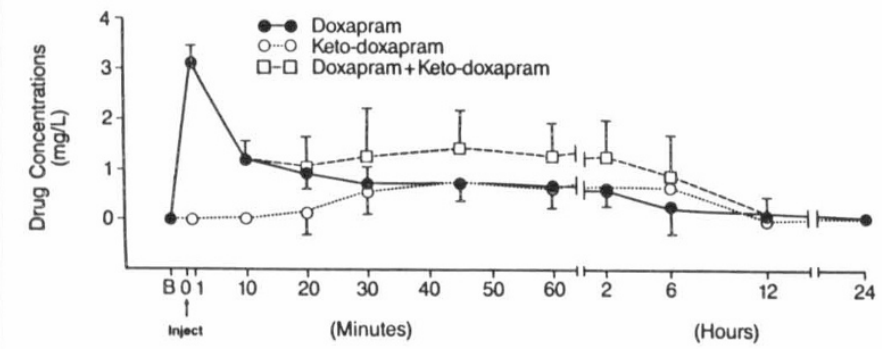

Fig. 6. Keto-dox generated over time from doxapram alone in the doxapram group. The closed circles represent absolute values of doxapram; the open circles represent keto-dox generated from doxapram. The squares represent the sum of keto-dox and doxapram when doxapram alone has been given. Corrections factors: doxapram $1 \mathrm{mg} / \mathrm{L}=$ $2.6 \mu \mathrm{mol} / \mathrm{L} ;$ keto-dox $1 \mathrm{mg} / \mathrm{L}=2.5 \mu \mathrm{mol} / \mathrm{L}$

Table 2. Pharmacokinetic profiles of keto-dox and doxapram*

\begin{tabular}{lll}
\hline \multicolumn{1}{c}{$\begin{array}{c}\text { Kinetic } \\
\text { variables }\end{array}$} & \multicolumn{1}{c}{ Keto-dox } & \multicolumn{1}{c}{ Doxapram } \\
\hline $\mathrm{t}_{1 / 2}$ alpha $(\mathrm{h})$ & $0.23(0.1-0.32)$ & $0.21(0.1-0.30)$ \\
$\mathrm{t}_{1 / 2}$ beta $(\mathrm{h})$ & $2.26(0.7-3.4)$ & $5.2(1.2-11.6) \dagger$ \\
$\mathrm{kel}\left(\mathrm{h}^{-1}\right)$ & $1.68(0.6-3.1)$ & $0.61(0.3-1.2)$ \\
$\mathrm{AUC}(\infty 24 \mathrm{~h})$ & $3.4(1.6-5.1)$ & $5.1(3.2-7.4)$ \\
$\mathrm{AVd}(\mathrm{L} / \mathrm{kg})$ & $1.1(0.34-2.05)$ & $1.2(0.5-2.0)$ \\
$\mathrm{CL}(\mathrm{L} / \mathrm{kg} / \mathrm{h})$ & $0.89(0.49-1.8)$ & $0.54(0.34-0.8)$ \\
$\mathrm{C}_{\max }(\mathrm{mg} / \mathrm{L})$ & $5.26 \ddagger(2.5-9.3)$ & $3.1 \ddagger(2.7-3.6)$ \\
\hline
\end{tabular}

*Values are means and range (in parentheses). $n=5$ lambs per group. kel, elimination rate constant; $\mathrm{AUC}$, area under the curve; $\mathrm{AVd}$, apparent volume of distribution; $\mathrm{CL}$, clearance; $\mathrm{C}_{\max }$, maximal plasma concentration obtained at $1 \mathrm{~min}$ of infusion.

$\dagger p=0.056$.

$\ddagger$ Correction factor: doxapram, $1 \mathrm{mg} / \mathrm{L}=2.6 \mu \mathrm{mol} / \mathrm{L}$; keto-dox, 1 $\mathrm{mg} / \mathrm{L}=2.5 \mu \mathrm{mol} / \mathrm{L}$.

[mean of $0.15 \mathrm{mg} / \mathrm{L}(0.37 \mu \mathrm{mol} / \mathrm{L})$ and range of 0.0 to $0.76 \mathrm{mg} /$ $\mathrm{L}(0.0$ to $1.9 \mu \mathrm{mol} / \mathrm{L})]$. Thereafter, the mean concentration of keto-dox was almost equivalent to that of doxapram during the period between $30 \mathrm{~min}$ and $2 \mathrm{~h}$ (Fig. 6).

Wide interindividual variability existed, however, for all drugs measured. Table 2 summarizes the different kinetic indices obtained in both the keto-dox and the doxapram groups. Keto-dox and doxapram exhibited a biexponential decay curve with a short alpha $t_{1 / 2}$ that was similar in both drugs, and a longer beta $t_{1 / 2}$ that was more prolonged in doxapram [mean of $5.2 \mathrm{~h}$ (range 1.211.6)] than in keto-dox [mean of $2.3 \mathrm{~h}$ (range 0.7-3.4)]. An example of the pharmacokinetic decay curves from one lamb of each group is presented in Fig. 7. Although the apparent volume of distribution was similar for both drugs, the total plasma clearance and the elimination rate constant were both slower for doxapram as compared with keto-dox.

\section{DISCUSSION}

Our study showed that keto-dox has respiratory stimulating properties in awake, unsedated newborn lambs. The changes in
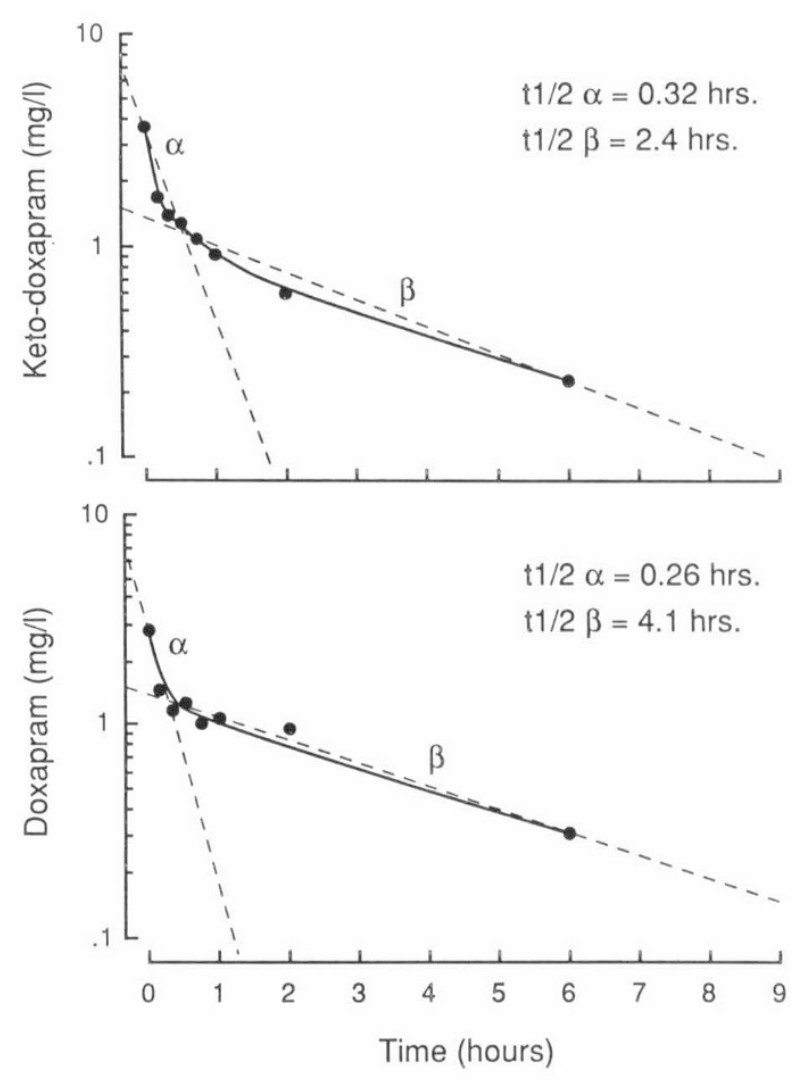

Fig. 7. Plasma disappearance curve of keto-dox and doxapram from one animal of each group. Top panel represents an example of keto-dox plasma disappearance curve in one animal; the bottom panel represents an example of doxapram plasma disappearance curve in another lamb. Corrections factors: doxapram $1 \mathrm{mg} / \mathrm{L}=2.6 \mu \mathrm{mol} / \mathrm{L}$; keto-dox $1 \mathrm{mg} / \mathrm{L}$ $=2.5 \mu \mathrm{mol} / \mathrm{L}$.

$\mathrm{f}$ and to a lesser extent those of $\mathrm{Vt}$ were responsible for the significant increase in $\mathrm{VE}$ and $\mathrm{Vt} / \mathrm{Ti}$, indicating, in turn, that keto-dox is a stimulant of respiratory drive. Our study also confirms that doxapram has potent respiratory stimulant effects $(1,2)$.

Comparable doses of keto-dox and doxapram produced ventilatory responses with similar patterns but significant differences in terms of magnitude and duration. It was the doxapram ventilatory response that was greater in both. These data also indicate that both respiratory stimulants keto-dox and doxapram have a short acting duration, but that the ventilatory effect of doxapram is more potent than that of keto-dox at the same dose; only doxapram is accompanied by adverse effects: an increase in blood pressure and a change in the animal's behavioral state. These adverse effects have already been noted with doxapram $(3,13)$.

In our study, we were unable to clearly establish the plasma concentrations of keto-dox and doxapram required to sustain these ventilatory effects. Because the ventilatory effects of ketodox occurred during the first minutes of injection (5 to $10 \mathrm{~min}$ ), it is probable that plasma concentrations of keto-dox between $5.3 \mathrm{mg} / \mathrm{L}(13.2 \mu \mathrm{mol} / \mathrm{L})$ [range 2.6 to $9.3 \mathrm{mg} / \mathrm{L}(6.5$ to 23.3 $\mu \mathrm{mol} / \mathrm{L})$, obtained at $1 \mathrm{~min}$ ] and $1.3 \mathrm{mg} / \mathrm{L}(3.25 \mu \mathrm{mol} / \mathrm{L})$ [range 1.1 to $1.7 \mathrm{mg} / \mathrm{L}(2.75$ to $4.2 \mu \mathrm{mol} / \mathrm{L})$, noted at $10 \mathrm{~min}$ ] are necessary to maintain respiratory stimulation. In contrast, inasmuch as the ventilatory effects of doxapram lasted for $20 \mathrm{~min}$ and were coincident with plasma concentrations at $1 \mathrm{~min}$ of 3.1 $\mathrm{mg} / \mathrm{L}(8.1 \mu \mathrm{mol} / \mathrm{L})$ [range 2.7 to $3.6 \mathrm{mg} / \mathrm{L}(7$ to $9.4 \mu \mathrm{mol} / \mathrm{L})$ ] and at $20 \mathrm{~min}$ of $0.88 \mathrm{mg} / \mathrm{L}(2.3 \mu \mathrm{mol} / \mathrm{L})$ [range 0.48 to $1.3 \mathrm{mg}$ ) $\mathrm{L}(1.2$ to $3.4 \mu \mathrm{mol} / \mathrm{L})$ ], a plasma doxapram concentration of 0.9 to $3.1 \mathrm{mg} / \mathrm{L}(2.3$ to $8.1 \mu \mathrm{mol} / \mathrm{L})$ appears to be necessary for maintenance of the ventilatory responses to doxapram. These estimated plasma drug concentrations may represent the mini- 
mal drug concentrations required to maintain ventilatory effects in newborn lambs.

In the doxapram group, the metabolite keto-dox was detected in the plasma only at $20 \mathrm{~min}$, suggesting that the early ventilatory effects produced are, in fact, related to doxapram per se. That supposition is further confirmed by the fact that the sum of plasma doxapram and its active metabolite concentration was not related to the maintenance ventilatory effects for more than $30 \mathrm{~min}$

In our study, keto-dox and doxapram exhibit biexponential decay curves, characterized by a short alpha $t_{1 / 2}$ and a longer beta $t_{1 / 2}$. And although the apparent volume of distribution was comparable, keto-dox had a faster elimination rate. These kinetic profiles, coupled with the relationship between ventilatory effects and drug concentrations, indicate that the ventilatory responses of both drugs appear to be related to the alpha phase. Therefore, the short duration of action of doxapram (20 to $30 \mathrm{~min}$ ) might be explained not only by its short alpha $t_{1 / 2}$, but also by its rapid biotransformation to keto-dox which, in turn, has a faster elimination rate than that of doxapram.

Doxapram is currently used for the treatment of apnea in newborn infants $(3-6,13)$. Our study demonstrates that its oxidative metabolite keto-dox is also a potent respiratory stimulant. We would suggest, however, that, until the role of ketodox on apnea is determined, it would be advisable to continue examining and accounting for the concentration of both drugs in the plasma.

In summary, both keto-dox and doxapram possess respiratory stimulant activities. Doxapram is more potent than keto-dox, but the latter seems to have fewer side effects, at least at the dosage level used in our study. Both drugs have a biexponential disappearance curve, but keto-dox has a faster elimination rate. Doxapram is also metabolized to keto-dox, but not vice versa. Those results are sufficiently encouraging to support further studies on keto-dox as a therapeutic agent.

Acknowledgments. The authors thank Drs. Michel Bureau and
Aurore Côté for their manuscript review, Rita Barrafato, Ginette Bérubé, and Line Lapointe for their secretarial support, and Christine Lalonde for her technical assistance.

\section{REFERENCES}

1. Funderbruk WH, Oliver KL, Ward JW 1965 Electrophysiologic analysis of the site of action of doxapram hydrochloride. J Pharmacol Exp Ther 151:360368

2. Hirsh K, Wang SC 1974 Selective respiratory stimulating action of doxapram compared to pentylenetetrazol. J Pharmacol Exp Ther 189:1-11

3. Barrington KJ, Finer NN, Peters KL, Barton J 1986 Physiologic effects of doxapram in idiopathic apnea of prematurity. J Pediatr 108:124-129

4. Bairam A, Vert P 1986 Low-dose doxapram for apnea of prematurity. Lancet 5:793-794

5. Eyal F, Alpan G, Sagi E, Glick B, PelefO, Djani Y, Arad I 1985 Aminophylline versus doxapram in idiopathic apnea of prematurity. Pediatrics 75:709-713

6. Alpan G, Eyal F, Sagi E, Springer C, Patz D, Goder K 1984 Doxapram in the treatment of idiopathic apnea of prematurity unresponsive to aminophylline. J Pediatr 104:634-637

7. Bairam A, Beharry K, Laudignon N, Rex J, Branchaud C, Papageorgiou A, Aranda JV 1989 Metabolism of doxapram in human fetal liver organ culture. Pediatr Res 25:64(abstr)

8. Robson RH, Prescott LF 1979 A pharmacokinetic study of doxapram in patients and volunteers. Br J Clin Pharmacol 7:81-87

9. Aranda JV, Mandelberg A, Beharry K, Rex J, Peleg O, Eyal F 1987 Metabolism of doxapram in premature newborns. Pediatr Res 21:232(abstr)

10. Bureau MA, Lamarche J, Foulon P, Zinman R, Dalle D 1985 Postnatal maturation of respiration in intact and carotid body denervated lambs. $\mathrm{J}$ Appl Physiol 59:869-874

11. Blanchard PW, Côté A, Hobbs S, Foulon P, Aranda JV, Bureau MA 1986 Abolition of ventilatory response to caffeine in chemodenervated lambs. J Appl Physiol 61:133-137

12. Bureau MA, Côté A. Blanchard PW. Hobbs S. Foulon P. Dalle D 1986 Exponential and diphasic ventilatory response to hypoxia in conscious lambs. J Appl Physiol 61:836-842

13. Hayakawa F, Hakamada S, Kuniyoshi K, Nakashima T, Miyashi Y 1986 Doxapram in the treatment of idiopathic apnea of prematurity: desirable dosage and serum concentrations. J Pediatr 109:138-140

14. Aranda JV, Beharry K, Rex J, Linder N, Blanchard PW 1988 High pressure liquid chromatographic microassay for simultaneous measurements of doxapram and its metabolites in premature newborn infants. J Liq Chromatogr 11:2983-2991

15. Sokal RR, Rolf FJ 1981 Biometry. In: The Principles and Practice of Statistics in Biological Research. Freeman, New York, p 254

16. Litovitz T 1986 The alcohols: ethanol, methanol, isopropanol, ethylene glycol. Pediatr Clin North Am 33:311-323 